\title{
Intracortical Functional Connectivity Predicts Arousal to Noxious Stimuli during Sleep in Humans
}

\author{
Hélène Bastuji, ${ }^{1,2}$ Andéol Cadic-Melchior, ${ }^{1}$ Michel Magnin, ${ }^{1}$ and Luis Garcia-Larrea ${ }^{1,3}$ \\ ${ }^{1}$ Central Integration of Pain (NeuroPain) Lab, Lyon Neuroscience Research Center, Institut National de la Santé et de la Recherche Médicale U1028, \\ Centre National de la Recherche Scientifique, UMR5292, Université Claude Bernard, Bron, F-69677, France, ${ }^{2}$ Centre du Sommeil et Service de \\ Neurologie Fonctionnelle et d'Épileptologie, Hospices Civils de Lyon, Bron, F-69677, France, and ${ }^{3}$ Centre d'évaluation et de traitement de la douleur, \\ Hôpital Neurologique, Lyon, 69677, France
}

Nociceptive stimuli disrupt sleep, but may, or may not, entail an arousal. While arousal reactions go along with the activation of a widespread cortical network, the factors enabling such activation remain unknown. Here we used intracranial EEG in humans to test the relation between the cortical activity immediately preceding a noxious stimulus and the capacity of such a stimulus to trigger arousal. Intracranial EEG signals were analyzed during all-night sleep in 14 epileptic patients (4 women), who received laser stimuli slightly above their individual pain threshold. During $5 \mathrm{~s}$ preceding each stimulus, the functional correlation (spectral phase-coherence) between the main spinothalamic sensory area (posterior insula) and 12 other brain regions, grouped in four networks, as well as their spectral contents, were contrasted according to the presence of a stimulus-induced arousal, and then fed into a logistic regression model to assess their predictive value. Enhanced prestimulus phase-coherence between the sensory posterior insula and neocortical and limbic areas increased significantly the probability of arousal to nociceptive stimuli, in both slow-wave (N2) and rapid eye movements/paradoxical sleep. Furthermore, during N2 sleep, arousal was facilitated by stimulus delivery in periods of attenuated slow-wave activity. Together, these data indicate that sleep micro-states with enhanced interareal communication facilitate information transfer from sensory to higherorder cortical areas, and hence physiological arousal.

Key words: nociceptive stimulus; sleep; arousal; intracerebral EEG; human

Significance Statement

Sleep is commonly subdivided into stages based on specific electrophysiological characteristics; however, within each single sleep stage, the functional state of the brain is continuously changing. Here we show that the probability for a phasic noxious stimulus to entail an arousal is modulated by the prestimulus interareal phase-coherence between sensory and higher-level cortical areas. Fluctuations in interareal communication immediately before the noxious stimulus may determine the responsiveness to incoming input by facilitating or preventing the transfer of noxious information from sensory to multiple higherlevel cortical networks.

\section{Introduction}

Being awakened by a noxious stimulus is an objective marker of its intrusiveness. During nocturnal sleep, stimuli disrupting homeostasis awake the subject in a very variable proportion of cases, from $30 \%$ for nociceptive pricking to $80 \%$ in the case of apneas (Lavigne et al., 2000, 2004; Bastuji et al., 2008; Chouchou

\footnotetext{
Received Nov. 19, 2020; revised Apr. 13, 2021; accepted Apr. 15, 2021

Author contributions: H.B., A.C.-M., and L.G.-L. designed research; H.B. and A.C.-M. performed research; H.B., A.C.-M., and L.G.-L. analyzed data; H.B., M.M., and L.G.-L. wrote the paper.

This work was supported by French Society for Pain Evaluation and Therapy SFETD Translational Research Grant 2012-14, LABEX CORTEX ANR-11-LABX-0042 and ANR-11-IDEX-0007), Region Rhone-Alpes/France ARC2 2012-2015 scholarship, and Institut National de la Santé et de la Recherche Médicale Interface Grant to H.B.

The authors declare no competing financial interests.

Correspondence should be addressed to Hélène Bastuji at bastuji@univ-lyon1.fr.

https://doi.org/10.1523/JNEUROSCI.2935-20.2021

Copyright $\odot 2021$ the authors
}

et al., 2014). Several lines of evidence suggest that the level of brain activation immediately before the stimulus may be crucial to modulate responsiveness to incoming input. During wakefulness, the prestimulus activity in different areas has been reported to correlate with the subsequent perception of nociceptive stimuli and/or the magnitude of pain ratings (Boly et al., 2007; Ploner et al., 2010; Ohara et al., 2008; Wiech et al., 2010). Transition from sleep to waking is preceded by signs of activation in multiple cortical areas (Magnin et al., 2010), and during sleep parietofrontal and cingulate activations preceded purpose-oriented motor responses (Mazza et al., 2014).

Our driving hypothesis in the present study was that the cerebral activity preceding a noxious stimulus delivered during sleep, and in particular the functional state of connectivity between sensory and higher-level areas, could largely determine the poststimulus neural responses, hence facilitating or impeding the 
Table 1. Individual clinical, MRI, and iEEG data

\begin{tabular}{|c|c|c|c|c|c|}
\hline Patient no. & Gender/age (yr) & Treatment (mg/day before-after tapering) & MRI & Seizure onset & No. of electrodes \\
\hline P1 & $M / 27$ & $\begin{array}{l}\text { Lamotrigine } 600-300 \\
\text { Carbamazepine } 1600-600\end{array}$ & $\mathrm{R}$ frontal dysplasia & $R$ frontal & $12 / R+2 / L$ \\
\hline P2 & $M / 19$ & Carbamazepine 1200-800 Valproate 1000-500 Clobazam 10-10 & R fronto-orbital & $R$ fronto-orbital & $11 / \mathrm{R}$ \\
\hline P3 & $\mathrm{F} / 23$ & $\begin{array}{l}\text { Levetiracetam } 2000-1000 \\
\text { Lamotrigine } 800-300\end{array}$ & L hippocampal atrophy & L mesial temporal & $11 / L$ \\
\hline P4 & $F / 18$ & $\begin{array}{l}\text { Oxcarbazepine } 600-0 \\
\text { Levetiracetam } 3000-0\end{array}$ & L temporal dysplasia & L parietal & $13 / L$ \\
\hline P5 & $F / 37$ & $\begin{array}{l}\text { Carbamazepine } 1600-600 \\
\text { Pregabalin 300-75 }\end{array}$ & Normal & L mesial temporal & $13 / L$ \\
\hline P6 & $F / 51$ & $\begin{array}{l}\text { Oxcarbazepine } 600-200 \\
\text { Clobazam 20-10 }\end{array}$ & Normal & R temporal & $12 / R$ \\
\hline P7 & $M / 20$ & $\begin{array}{l}\text { Valproate } 1500-500 \\
\text { Carbamazepine } 1200-600 \\
\text { Levetiracetam 2000-1000 }\end{array}$ & L temporal atrophy & L temporal & $13 / L$ \\
\hline P8 & $M / 26$ & $\begin{array}{l}\text { Carbamazepine } 1000-200 \\
\text { Lamotrigine } 400-200 \text { Pregabalin 300-75 }\end{array}$ & L hippocampal atrophy & L mesial temporal & $12 / L$ \\
\hline P9 & $M / 20$ & $\begin{array}{l}\text { Levetiracetam } 3000-0 \\
\text { Zonisamide } 300-0\end{array}$ & R hippocampal atrophy & $\mathrm{R}$ mesial temporal & $12 / R$ \\
\hline P10 & $M / 21$ & $\begin{array}{l}\text { Topiramate } 300-200 \\
\text { Oxcarbazepine } 900-400 \\
\text { Lamotrigine } 400-100\end{array}$ & $\mathrm{R}$ temporal dysplasia & R temporal & $11 / R+3 / L$ \\
\hline P11 & $M / 39$ & $\begin{array}{l}\text { Lamotrigine } 400-200 \\
\text { Topiramate } 300-200 \\
\text { Levetiracetam } 3000-1000 \\
\text { Lacosamide } 200-100\end{array}$ & L hippocampal atrophy & L mesial temporal & $11 / L$ \\
\hline P12 & $\mathrm{M} / 19$ & Carbamazepine 600-0 & Normal & R mesial temporal & $11 / R$ \\
\hline P13 & $M / 32$ & $\begin{array}{l}\text { Levetiracetam 1500-1000 } \\
\text { Oxcarbazepine } 750-150\end{array}$ & L hippocampal atrophy & L basal temporal & $13 / L+2 / R$ \\
\hline P14 & $M / 37$ & $\begin{array}{l}\text { Carbamazepine } 800-400 \\
\text { Topiramate } 400-200 \\
\text { Clobazam } 10-5\end{array}$ & Normal & L perisylvian & $13 / L$ \\
\hline
\end{tabular}

subject's awakening. To test this hypothesis, we used intracranial electroencephalographic (iEEG) recordings in humans to analyze neural activity in both time-frequency and functional connectivity (phase-coherence) domains during the $5 \mathrm{~s}$ preceding the delivery of noxious stimuli, and correlated them with the occurrence of an arousal reaction.

\section{Subjects and Methods}

Patient selection. Fourteen patients with refractory partial epilepsy were included in the study (10 men, 4 women; mean age 28 years, range 19-51 years). They were consecutive patients agreeing to participate in the study, in accord with their physician in charge and having at least one electrode contact in the posterior insula together with contacts in areas belonging to at least two other networks (see below). To delineate the extent of the cortical epileptogenic area and to plan a tailored surgical treatment, depth EEG recording electrodes (diameter $0.8 \mathrm{~mm}$; 5-15 recording contacts $2 \mathrm{~mm}$ long, intercontact interval $1.5 \mathrm{~mm}$ ) were implanted according to the Talairach space (Guénot et al., 2001; Isnard et al., 2018). The procedure aims at recording spontaneous seizures but also includes the functional mapping of potentially eloquent cortical areas using evoked potentials recordings and cortical electrical stimulation (Ostrowsky et al., 2002; Mazzola et al., 2006). In agreement with French regulations relative to invasive investigations with direct individual benefit, patients were fully informed about electrode implantation, iEEG and evoked potential recordings, and cortical stimulation procedures used to localize the epileptogenic cortical areas, and gave their consent. The laser stimulation paradigm was approved by the local and regional Ethics Committee (CPP Sud Est IV no. 2006-A00572-49).

Spinothalamic-specific laser stimulations were performed during one full night after a minimal delay of $5 \mathrm{~d}$ after electrode implantation; at that time, any "first-night" effect had faded away, and antiepileptic drugs had been stopped or tapered down with daily small doses (Table 1).
None of these patients reported pain symptoms before or after the recording session.

Electrode implantation. Intracerebral electrodes were implanted using the Talairach's stereotactic frame (Talairach and Bancaud, 1973). A cerebral angiography was performed in stereotactic conditions using an X-ray source located $4.85 \mathrm{~m}$ away from the patient's head. This eliminates the linear enlargement because of X-ray divergence and allows a 1:1 scale so that films could be used for measurements without any correction. In a second step, the relevant targets were identified on the patient's MRI, previously enlarged to a scale of 1:1. As MR and angiographic images were at the same scale, they could easily be superimposed, so as to avoid damage to blood vessels and minimize the risk of hemorrhage during electrode implantation.

Anatomical localization of recording sites. The localization of recording contacts was determined using two different procedures. In 7 patients implanted before the year 2010, MRI could not be performed with electrodes in place because of the physical characteristics of the stainless-steel contacts. In these cases, the scale 1:1 postimplantation skull radiographs performed within the stereotactic frame were superimposed to the preimplantation scale 1:1 MRI slice corresponding to each electrode track, thus permitting to plot each contact onto the appropriate MRI slice of each patient to determine its coordinates (MRIcro software) (Rorden and Brett, 2000). In the other 7 patients, the implanted electrodes were MRI-compatible and cortical contacts could be directly visualized on the postoperative 3D MRIs. In both cases, anatomic scans were acquired on a 3-Tesla Siemens Avanto Scanner using a 3D MPRAGE sequence with the following parameters: TI/TR/TE 1100/2040/2.95 ms, voxel size: $1 \times 1 \times 1 \mathrm{~mm}^{3}$, FOV $=256 \times 256 \mathrm{~mm}^{2}$.

Intracortical electrode contacts were mapped to the standard stereotaxic space (MNI) by processing MRI data with Statistical Parametric Mapping (SPM12, Wellcome Department of Cognitive Neurology; http://www.fil.ion. ucl.ac.uk/spm/). Anatomical T1-3D images before and after implantation were coregistered and normalized to the MNI template brain image using a 
A

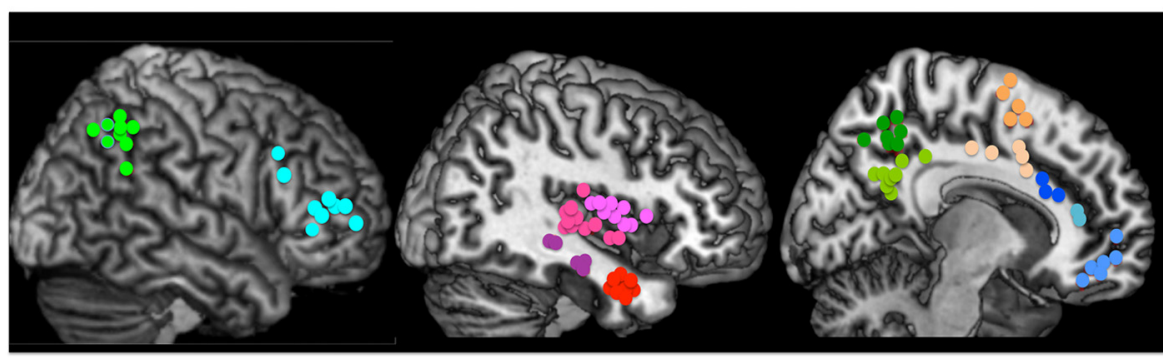

B

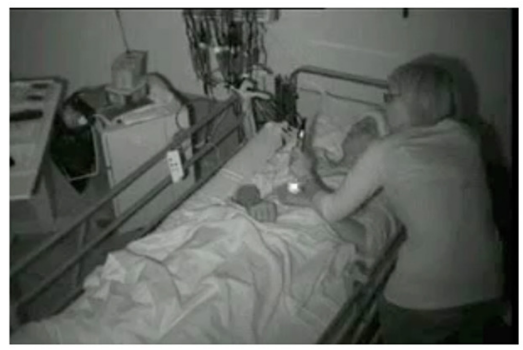

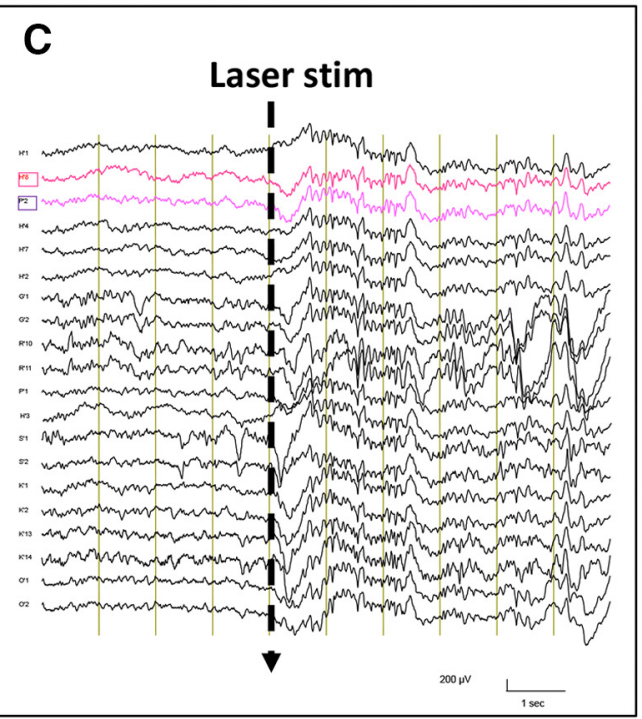
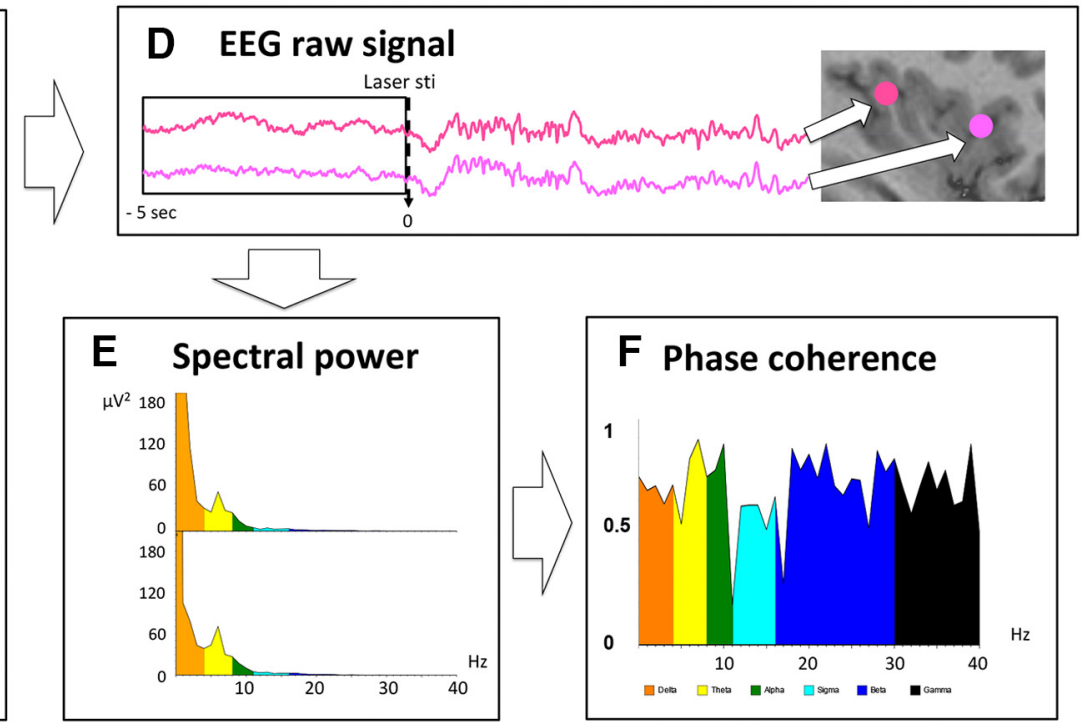

Figure 1. A, Localization of recording contacts used for analysis in each area represented on MNI brain templates. Left, Brain convexity with contacts in PPC $(n=9)$, dIPFC $(n=9)$. Middle, Sagittal slice with contacts in posterior insula $(n=14)$, anterior insula $(n=9)$, hippocampus $(n=5)$, amygdala $(n=9)$. Right, Mid-sagittal slice with contacts in precuneus $(n=7)$, posterior cingulate cortex $(n=8)$, mid cingulate cortex $(n=5)$, supplementary motor area $(n=5), \operatorname{ACC}(n=3)$, perigenual ACC $(n=2)$, orbitofrontal cortex $(n=6)$. $\boldsymbol{B}$, Photography of a patient being stimulated with the laser during sleep. $\boldsymbol{C}$, Example of iEEG traces before and after a laser stimulus. D, Two iEEG signals obtained before and after the laser stimulus: one in the posterior insula (magenta) and the other in the anterior insula (pink). On the MRI slice, magenta and pink circles represent the localization of these two contacts in the insula. $\boldsymbol{E}$, Spectral powers of both iEEG signals during the $5 \mathrm{~s}$ before the stimulus. $\boldsymbol{F}$, Phase-coherence between the posterior insula signal and the one simultaneously recorded in the anterior insula.

mutual information approach and the segmentation module of SPM12, which segments, corrects bias, and spatially normalizes images with respect to the MNI model. Then, the cortical localization of electrodes was performed using a regional atlas (WFU Pickatlas version 3) in MRIcron ${ }^{\circ}$. In the 7 patients with MRI-compatible electrodes, the cortical contacts could be directly visualized on the postoperative normalized 3D MRIs. In the 7 patients without MRI-compatible electrodes, the coordinates of contacts were determined on their own preoperative MRI according to the procedure described above, thus permitting to plot each contact onto the appropriate MRI slice of each patient (MRIcron software) to determine its MNI coordinates. Figure $1 A$ illustrates the 91 cerebral contacts from which iEEG signal was recorded and analyzed.

Nociceptive-specific laser stimulation. Radiant nociceptive heat pulses of $5 \mathrm{~ms}$ duration were delivered with a Nd:YAP-laser (Yttrium Aluminum Perovskite; wavelength $1.34 \mu \mathrm{m}$ ). The laser beam was transmitted from the generator to the stimulating probe via an optical fiber of $10 \mathrm{~m}$ length (550 $\mu \mathrm{m}$ diameter with sub-miniature version A-905 connector). Perceptive and nociceptive thresholds were determined in each patient immediately before the recording session. Nociceptive thresholds to A $\delta$ stimuli were determined as the minimal laser energy producing a pricking sensation, compared with pulling a hair or receiving a boiling water drop in at least two of three stimuli. They were obtained in all subjects with energy densities between 80 and $100 \mathrm{~mJ} / \mathrm{mm}^{2}$, which are within the usual data range observed in our laboratory and those reported by others using Nd:YAP lasers (Cruccu et al., 2008). These parameters have been validated as being able to activate selectively the spinothalamic system in humans (e.g., Garcia-Larrea et al., 2010; Perchet et al., 2012; La Cesa et al., 2018), including during sleep, where they arouse subjects in $\sim 30 \%$ of the cases (Bastuji et al., 2008; Mazza et al., 2012).
Data acquisition and recording procedure. In each patient, there were two runs of 10-15 stimulations each. Interstimulus interval was pseudo-randomly adjusted online and varied between 10 and $20 \mathrm{~s}$. Stimulus energy was set at the individual nociceptive threshold previously determined and applied to the skin in the superficial radial nerve territory, on the dorsum of the hand contralateral to the hemispheric side of electrode implantation. The heat spot was slightly shifted over the skin surface between two successive stimuli to avoid both sensitization and peripheral nociceptor fatigue. Eight patients were stimulated on the right hand, and 6 on the left hand. Then the patients were allowed to sleep at their own time, and no further laser stimulation was delivered until a minimum of $20 \mathrm{~min}$ of continuous sleep had been obtained. The identification of the different sleep stages (N2, N3, and PS) was done online by one of the investigators (H.B.) expert in sleep studies. If one stimulus awoke the sleeper, the stimulation sequence was immediately discontinued. A second investigator entered the room and delivered nociceptive pulses transmitted through the optic fiber from the laser stimulator. The $10 \mathrm{~m}$ optical fiber transited under the door separating the recording and sleeping areas and allowed to stimulate conveniently the dorsum of the hand despite movements of the subjects during the night. Both the sleeping subject and the investigator wore eye protections. Runs of stimulations were delivered during the different sleep stages up to 6:00 A.M. Recordings were performed during all night in referential mode, the reference electrode being chosen for each patient on an implanted contact located in the skull. The iEEG signal was recorded continuously from 96 to 128 channels at a sampling frequency of 256 or $512 \mathrm{~Hz}$, amplified and band pass filtered $(0.33-128 \mathrm{~Hz} ;-3 \mathrm{~dB}$, $12 \mathrm{~dB}$ /octave) to be stored in hard disk for offline analysis (Micromed SAS). 
Table 2. MNI coordinates $(x, y, z)$ of cerebral contacts

\begin{tabular}{|c|c|c|c|c|c|c|c|c|c|c|c|c|c|}
\hline Contacts & $\begin{array}{l}\text { Posterior } \\
\text { insula }(n=14)\end{array}$ & $\begin{array}{l}\text { DLPFC } \\
(n=9)\end{array}$ & $\mathrm{PPC}(n=9)$ & $\begin{array}{l}\text { Anterior insula } \\
(n=9)\end{array}$ & $\begin{array}{l}\text { ACC } \\
(n=3)\end{array}$ & $\begin{array}{l}\text { Mid-cingulate } \\
\text { cortex }(n=5)\end{array}$ & $\begin{array}{l}\text { Supplementary motor } \\
\text { area }(n=5)\end{array}$ & $\begin{array}{l}\text { Posterior cingulate } \\
\text { cortex } n=8 \text { ) }\end{array}$ & $\begin{array}{l}\text { Precuneus } \\
(n=7)\end{array}$ & $\mathrm{pACC}(n=2)$ & $\begin{array}{l}\text { Hippocampus } \\
(n=5)\end{array}$ & $\begin{array}{l}\text { Orbitofrontal } \\
\text { cortex }(n=6)\end{array}$ & $\begin{array}{l}\text { Amygdala } \\
(n=9)\end{array}$ \\
\hline \multicolumn{14}{|l|}{ Patients } \\
\hline P1 & $38,-6,4$ & $52,32,19$ & $43,-51,52$ & $33,24,6$ & $5,32,18$ & $7,-7,38$ & $5,3,70$ & & $5,-51,52$ & & & $6,41,-13$ & \\
\hline P2 & $37,-20,5$ & & $52,-48,46$ & & $4,29,22$ & & & $5,-48,18$ & $5,-48,46$ & & & & $21,-3,-18$ \\
\hline P3 & $37,-24,2$ & & $45,-55,41$ & & & & & $3,-48,23$ & $8,-55,41$ & & & & $20,-6,-23$ \\
\hline P4 & $38,-2,-5$ & $49,20,34$ & $48,-60,38$ & $34,-4,11$ & & $4,20,34$ & $6,0,64$ & $2,-30,33$ & $8,-66,44$ & & $24,-1,-28$ & & \\
\hline P5 & $35,-23,5$ & $50,22,23$ & $37,-53,45$ & $33,6,12$ & & $4,22,28$ & $3,8,57$ & $4,-50,20$ & & & & $5,46,-14$ & \\
\hline P6 & $37,-1,-4$ & & & $38,16,1$ & & & & $10,-45,25$ & & & $22,-15,-25$ & & $15,-6,-20$ \\
\hline P7 & $38,-16,17$ & $38,41,6$ & $39,-55,50$ & $35,0,11$ & & & $8,12,51$ & & $3,-57,49$ & & & $6,53,-9$ & $18,-3,-20$ \\
\hline P8 & $33,-23,5$ & $30,44,11$ & & $32,2,8$ & $5,44,14$ & $6,4,36$ & $6,4,52$ & & & & & & \\
\hline P9 & $35,-23,2$ & $37,50,9$ & $43,-58,49$ & $33,11,9$ & & & & & $12,-57,52$ & & & $6,37,-19$ & $16,-3,-22$ \\
\hline P10 & $38,-23,9$ & & $43,-58,49$ & & & $7,17,37$ & & $8,-57,25$ & & & $29,-18,-16$ & & \\
\hline P11 & $36,-25,1$ & & $39,-60,45$ & & & & & $9,-52,26$ & $14,-63,45$ & & $29,-31,-6$ & & $21,-9,-26$ \\
\hline P12 & $35,-22,7$ & $51,37,21$ & & $36,11,2$ & & & & & & $7,44,10$ & $27,-16,-20$ & & $25,2,-22$ \\
\hline P13 & $36,-12,-1$ & $40,36,0$ & & & & & & $6,-42,31$ & & & & $4,54,1$ & $17,0,-11$ \\
\hline P14 & $37,-12,2$ & $42,48,9$ & & $35,8,5$ & & & & & & $8,46,7$ & & $7,48,-11$ & $24,-2,-21$ \\
\hline Mean & $36,-17,4$ & $43,37,15$ & $43,-55,46$ & $34,8,7$ & $5,35,18$ & $6,11,35$ & $6,5,59$ & $6,-47,25$ & $8,-57,47$ & $7.5,45,8$ & $26,-16,-19$ & $6,47,-11$ & $20,-3,-20$ \\
\hline SD & $2,9,6$ & $8,11,10$ & $5,4,4$ & $1,2,2$ & $1,8,4$ & $2,12,4$ & $2,5,8$ & $3,8,5$ & $4,6,4$ & $1,1,2$ & $3,11,9$ & $1,7,7$ & $3,3,4$ \\
\hline
\end{tabular}

\section{Pre-stimulus phase coherence between sensory insula and cerebral networks}

A

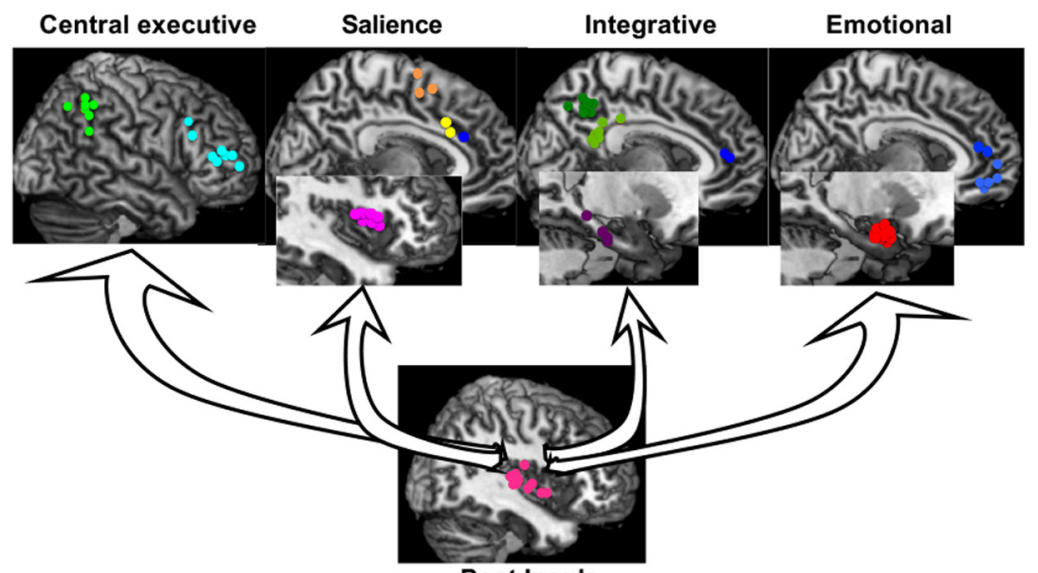

B

N2 sleep

Post Insula

Paradoxical sleep

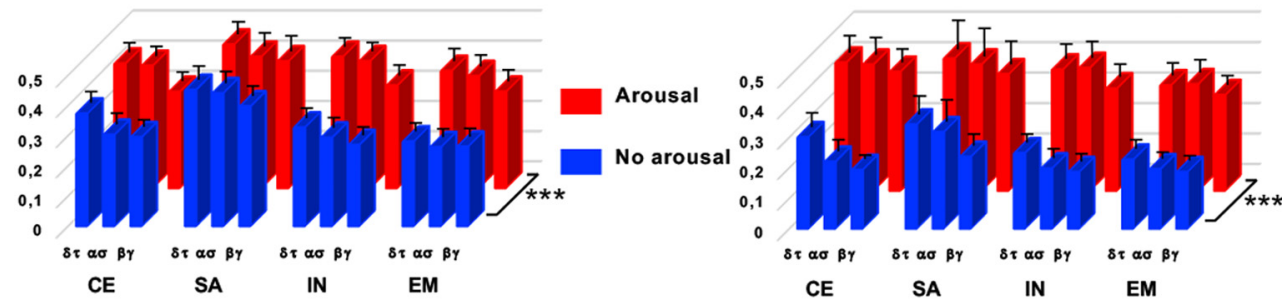

Figure 2. Phase-coherence between posterior insula and the other areas, grouped in four networks. A, Bottom, Posterior insula. Top, Left to right, (entral executive (dIPFC, PPC); salience (anterior insula, mid cingulate, supplementary motor areas, anterior cingulate); integrative (posterior cingulate, precuneus, hippocampus, perigenual cingulate); emotional (amygdala, orbitofrontal, perigenual cingulate) networks. $\boldsymbol{B}$, Mean phase-coherence levels ( \pm SEM) of iEEG frequency bands between posterior insula and the four networks calculated in the $5 \mathrm{~s}$ preceding nociceptive stimuli for N2 sleep (left) and PS (right). Phase-coherence values in case of arousal (red) and nonarousal (blue). CE, Central executive; SA, salience; IN, integrative; EM, emotional. Phase-coherence values are higher in case of arousal than in nonarousal conditions. $* * * p<0.0001$.

Sleep scoring and arousal reactions. Criteria of the American Academy of Sleep Medicine adapted to intracerebral recordings (Magnin et al., 2004; Bastuji et al., 2012; Claude et al., 2015) were used for iEEG data. Hypnograms based on $30 \mathrm{~s}$ epochs allowed determining the vigilance state during which stimuli were delivered. As already observed in previous studies, laser stimuli delivered during sleep phase N3 induced quite systematically a shift to N2 sleep stage (Bastuji et al., 2008, 2012); therefore, only recordings from sleep stage N2 and PS are presented here. Following the American Academy of Sleep Medicine
Table 3. Post hoc analyses of phase-coherence values with respect to the three frequency bands in N2 sleep and PS ${ }^{a}$

\begin{tabular}{lll}
\hline Frequency band & N2 sleep & PS \\
\hline$\delta-\theta$ vs $\alpha-\sigma$ & $t_{(91)}=3.22 ; p=0.0018 ; d=0.19$ & $t_{(77)}=2.15 ; p=0.035 ; d=0.13$ \\
$\delta-\theta$ vs $\beta-\gamma$ & $t_{(91)}=7.22 ; p<0.0001 ; d=0.50$ & $t_{(77)}=5.44 ; p<0.0001 ; d=0.36$ \\
$\alpha-\sigma$ vs $\beta-\gamma$ & $t_{(91)}=5.24 ; p<0.0001 ; d=0.30$ & $t_{(77)}=4.23 ; p=0.0001 ; d=0.20$ \\
\hline
\end{tabular}

${ }^{a} t, p$, and $d$ values if significant. 
Table 4. Post hoc analyses of phase-coherence values with respect to the significant interaction between arousal and frequency bands in N2 sleep and PS ${ }^{a}$

\begin{tabular}{lllll}
\hline Frequency band & N2 arousal & N2 nonarousal & PS arousal & PS nonarousal \\
\hline$\delta-\theta$ vs $\alpha-\sigma$ & $t_{(45)}=1.46 ; p 0.152$ & $t_{(45)}=3.28 ; p 0.004 ; d=0.27$ & $t_{(38)}=0.07 ; p=0.944$ & $t_{(38)}=2.52 ; p=0.0318 ; d=0.35$ \\
$\delta-\theta$ vs $\beta-\gamma$ & $t_{(45)}=5.31 ; p<0.0001 ; d=0.60$ & $t_{(45)}=5.15 ; p<0.0001 ; d=0.43$ & $t_{(38)}=3.62 ; p=0.0017 ; d=0.26$ & $t_{(38)}=4.13 ; p=0.0006 ; d=0.62$ \\
$\alpha-\sigma$ vs $\beta-\gamma$ & $t_{(45)}=5.69 ; p<0.0001 ; d=0.46$ & $t_{(45)}=1.81 ; p 0.077$ & $t_{(38)}=4.02 ; p=0.0008 ; d=0.25$ & $t_{(38)}=2.04 ; p=0.0484 ; d=0.21$ \\
\hline
\end{tabular}

${ }^{a} t, p$, and $d$ values if significant.

criteria adapted for intracerebral recordings (Bastuji et al., 2012), “cortical arousals" were defined as bursts of waking cortical activity lasting at least $3 \mathrm{~s}$, and labeled "awakenings" if they persisted $>15 \mathrm{~s}$. These arousal reactions (cortical arousal and awakening) were considered as stimulusrelated if they occurred within $10 \mathrm{~s}$ after noxious stimulus onset. Signals obtained when stimuli were delivered during an arousal period were rejected.

Interareal functional connectivity. The main steps of iEEG signal processing are illustrated in Figure $1 C-F$. Using Brain Analyzer software, FFT of the $5 \mathrm{~s}$ iEEG signals preceding the stimuli was applied in three spectral bands: $\delta-\theta(0.5-7.5 \mathrm{~Hz}), \alpha-\sigma(8-15 \mathrm{~Hz})$, and $\beta-\gamma(16-40 \mathrm{~Hz})$, which were labeled "low," "intermediate," and "high" frequency bands, respectively. Higher $\gamma$ frequencies were not included, as they are less involved in long-range coherence relative to lower frequencies (von Stein and Sarnthein, 2000) and are often "nested" within slower oscillations (Lisman and Jensen, 2013). In each patient, iEEG spectral content was computed for all the brain contacts explored, during both sleep N2 and PS, and stored separately according to the presence (A) or the absence (NA) of an arousal or an awakening reaction after the stimulus. All contacts located within the epileptic network and/or in lesional tissues were excluded from the analysis.

Functional connectivity was studied using iEEG phase-coherence analysis, the sensory posterior insula acting as "seed region" with respect to the rest of the cerebral areas analyzed, grouped in four networks (MNI coordinates in Table 2). The posterior insula was chosen as the reference area, since this region receives the most important sensory projections from the spinothalamic system (Dum et al., 2009) and exhibits the earliest cortical nociceptive signals in humans (Frot and Mauguière, 2003; Bastuji et al., 2016). Networks selected for phase-coherence analysis were extracted following consensual literature on functional commonalities, and comprised the following: (1) a central "executive" network, including dorsolateral PFC (dlPFC) and posterior parietal cortex (PPC) areas; (2) a "salience" network, including the anterior insula, ACC, mid-cingulate cortex, and supplementary motor areas; (3) a "late-integrative network," including the posterior cingulate-precuneus, the perigenual cingulate cortex, and hippocampus; and (4) an "emotional" network joining orbitofrontal cortex, amygdala, and, again, the perigenual cingulate cortex (Neugebauer et al., 2009; Doucet et al., 2019). Phase-coherence was computed after FFT of the $5 \mathrm{~s}$ signal, in referential mode, for each spectral band power. The phase-coherence value was calculated as the quotient between correlation and autocorrelation for each frequency and each channel pair and underwent Fisher's $z$ transformation before statistical analysis, to transform the sampling distribution of coherence values so that it becomes normally distributed (Nunez et al., 1997). Coherence values were computed by calculating the cross-spectrum (a measure of the joint spectral properties of the two channels) normalized by their auto-spectrum (the power spectrum of each channel), as follows:

$$
\operatorname{Coh}\left(c_{1}, c_{2}\right)(f)=\frac{\left|\operatorname{Cov}\left(c_{1}, c_{2}\right)(f)\right|^{2}}{\left|\operatorname{Cov}\left(c_{1}, c_{1}\right)(f)\right|\left|\operatorname{Cov}\left(c_{2}, c_{2}\right)(f)\right|}
$$

with

$$
\operatorname{Cov}\left(c_{1}, c_{2}\right)(f)=\Sigma\left(c_{1, i}(f)-\operatorname{moy}\left(c_{1}(f)\right)\right)\left(c_{2, i}(f)-\operatorname{moy}\left(c_{2}(f)\right)\right)
$$

In the second formula, totaling is conducted using the segment number $i$. The average is obtained with reference to the segment with a fixed frequency $f$ and a fixed channel $c$.
Table 5. Post hoc analyses of the frequency bands with respect to spectral power in N2 sleep and PS ${ }^{a}$

\begin{tabular}{l}
\hline Frequency band N2 \\
\hline$\delta-\theta$ vs $\alpha-\sigma \quad t_{(177)}=33.39 ; p<0.0001 ; d=4.03 \quad t_{(183)}=45.02 ; p<0.0001 ; d=5.32$ \\
$\delta-\theta$ vs $\beta-\gamma \quad t_{(177)}=41.83 ; p<0.0001 ; d=4.62 \quad t_{(183)}=43.68 ; p<0.0001 ; d=5.39$ \\
$\alpha-\sigma$ vs $\beta-\gamma \quad t_{(177)}=8.48 ; p<0.0001 ; d=0.74 \quad t_{(183)}=3.25 ; p=0.0014 ; d=0.21$ \\
\hline${ }^{a} t, p$, and $d$ values if significant.
\end{tabular}

Table 6. Post hoc analyses of spectral power values with respect to the significant interaction between arousal and frequency bands in N2 sleep and PS ${ }^{a}$

\begin{tabular}{lll}
\hline Frequency band & N2 A vs NA & PS A vs NA \\
\hline$\delta-\theta$ & $t_{(89)}=3.27 ; p=0.0046 ; d=0.61$ & $t_{(91)}=3.00 ; p=0.0091 ; d=0.26$ \\
$\alpha-\sigma$ & $t_{(89)}=2.43 ; p=0.051$ & $t_{(91)}=0.34 ; p=0.93 ;$ not significant \\
$\beta-\gamma$ & $t_{(89)}=1.34 ; p=0.45$ & $t_{(91)}=0.13 ; p=0.93$ \\
\hline
\end{tabular}

${ }^{a} t, p$, and $d$ values if significant.

Statistical analyses. During N2 sleep, individual phase-coherence values between posterior insula and the different brain networks were submitted to a three-way mixed design ANOVA with "arousal" (yes/no) and "frequency band" (low, intermediate, high) as repeated measures ("within" factors), and the four cerebral networks as between factor. A similar ANOVA was performed with phase-coherence values obtained during PS. Post hoc tests (Holm-Sidak test corrected for multiple comparisons) were applied in case of significant effects following ANOVAs. The reported effect size measure was $\eta^{2}$ in ANOVA and Cohen's $d$ in $t$ tests.

Spectral power was normalized by dividing each value by the average of all spectrum values in a given patient, and this for each network and sleep stage. Individual spectral power values obtained during N2 sleep were submitted to a three-way mixed ANOVA with "arousal" and "frequency band" as within factors and "cerebral network" as between factor. The latter factor included the four previous networks and the posterior insula. A similar ANOVA was performed with power values obtained during PS. Post hoc tests (Holm-Sidak test corrected for multiple comparisons) were applied in case of significant effects following ANOVAs. The reported effect size measure was $\eta_{\mathrm{p}}^{2}$ for ANOVA and Cohen's $d$ for $t$ tests.

A logistic regression model was used to investigate to what extent a combination of explanatory variables would classify iEEG prestimulus periods as predicting arousal versus nonarousal. The default variables introduced in the model were those having shown a significant individual association with the two outcomes in ANOVA, on the condition that there were not themselves mutually correlated.

Statistical analyses were performed with GraphPad Prism 8 and StatView softwares.

\section{Results}

The prestimulus iEEG signals of the 14 patients with refractory partial epilepsy were obtained from 107 arousal and 185 nonarousal prestimulus segments during N2 sleep, and from 104 arousal and 215 nonarousal prestimulus segments during paradoxical sleep. The mean latency of arousals triggered by the stimulus was $1.19 \pm 0.07 \mathrm{~s}$ in sleep N2 and $0.95 \pm 0.06 \mathrm{~s}$ in PS. The mean interstimulus interval was $29.4 \pm 10.7 \mathrm{~s}$, and the $\mathrm{N}-1$ to $\mathrm{N}$ 


\section{Pre-stimulus spectral power}

A
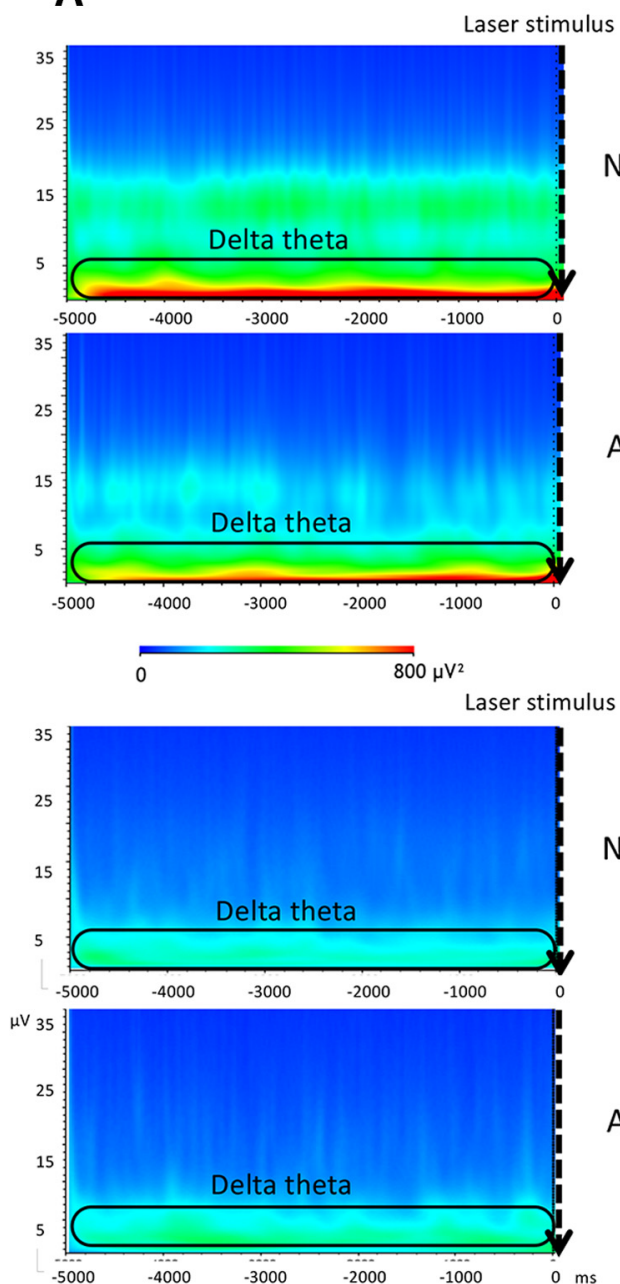

B

\section{N2 sleep}

No arousal

Arousal

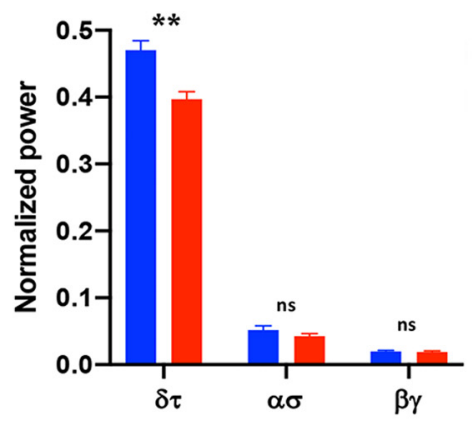

Paradoxical sleep

Arousal

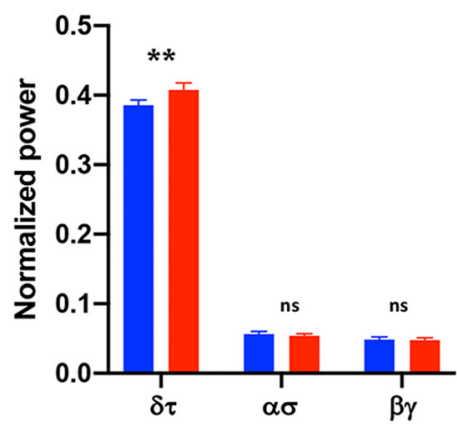

Figure 3. A, Grand averages of prestimulus time-frequency signals of all cerebral areas in sleep N2 and PS according to the absence (top) or presence (bottom) of an arousal after the stimulus. $\boldsymbol{B}$, Mean spectral powers ( \pm SEM) of the three frequency bands in the two sleep stages according to the presence (red) or absence (blue) of an arousal. In N2 sleep, $\delta$ - $\theta$ power was significantly decreased in case of arousal, while it was significantly increased in PS. $* * p<0.001$, ns, not significant.

interval was the same whether the $\mathrm{N}$ stimulus gave rise or not to an arousal ( $29 \pm 11.6$ vs $28.9 \pm 10.7 ; t=0.04 ; p=0.96)$.

To investigate whether arousal induced by one stimulus influenced the response to the following stimulus, we compared the probability of arousal to a stimulus $\mathrm{N}$ as a function of the response to the previous stimulation $(\mathrm{N}-1)$. In sleep $\mathrm{N} 2$, arousals triggered by a stimulus $\mathrm{N}$ represented $53 \%$ of the cases occurring after a preceding arousal-inducing stimulus, versus $47 \%$ after a nonarousing stimulus (diff \pm CI $95 \%=0.06 \pm 0.19$, not significant). In PS, these proportions were $48 \%$ versus $52 \%$, respectively $($ diff + CI $95 \%=0.04 \pm 0.14$, not significant $)$.

\section{Phase-coherence}

During N2 sleep, phase-coherence levels between sensory posterior insula and the four networks were significantly enhanced when preceding an arousal reaction, in contrast to no arousal $\left(0.40 \pm 0.01\right.$ vs $0.32 \pm 0.01 ; F_{(1,42)}=19.99$; $\left.p<0.0001 ; \eta_{p}^{2}=0.31\right)$. There was also a significant effect of frequency band $\left(F_{(2,42)}=28.21 ; p<0.0001 ; \eta_{p}^{2}=0.40\right)$, but not of cerebral network $\left(F_{(3,42)}=2.42 ; p=0.08\right)$. Figure 2 summarizes phase-coherence values for the different frequency bands in each condition and network. On post hoc testing, internetwork phase-coherence preceding arousal was significantly higher for low frequencies $(\delta-\theta)$ compared with intermediate and high frequencies $(\alpha-\sigma ; \beta-\gamma)$, and also higher for $\alpha-\sigma$ compared with $\beta-\gamma$ (Table 3 ). There was a small significant interaction between arousal and frequency band $\left(F_{(6,84)}=3.30 ; p=0.042 ; \eta_{p}^{2}=0.07\right)$ (Table 4$)$.

During PS, ANOVA showed a similar set of significant effects: phase-coherence between posterior insula and all other networks was higher before arousal compared with no arousal $\left(0.374 \pm 0.015\right.$ vs $0.224 \pm 0.010 ; F_{(1,35)}=15.63 ; p=0.0004 ; \eta_{p}^{2}=$ $0.31)$. There was also a significant effect of frequency band $\left(F_{(2,35)}=11.18 ; p<0.0001 ; \eta_{p}^{2}=0.24\right)$, without differences among cerebral networks $\left(F_{(3,35)}=1.11 ; p=0.36\right)$. Figure 2 summarizes phase-coherence values for the different frequency bands in each condition and network. Phase-coherence was significantly higher for low $(\delta-\theta)$ compared with intermediate and high frequencies $(\alpha-\sigma$ and $\beta-\gamma)$, and also higher for $\alpha-\sigma$ relative to $\beta-\gamma$ (Table 3 ). There was a significant interaction between arousal and frequency band $\left(F_{(2,70)}=4.31 ; p=0.0172\right.$; $\left.\eta_{p}^{2}=0.11\right)$, explained by phase-coherence differences between low and intermediate bands being nonsignificant in the arousal condition (Table 4). 


\section{Spectral power}

Spectral power during N2 sleep was significantly lower preceding an arousal reaction $\left(F_{(1,84)}=12.69 ; p=0.0006 ; \eta_{p}^{2}=0.13\right)$, without significant variation across the different cerebral networks $\left(F_{(4,84)}=0.15 ; p>0.999\right)$. The spectral power also differed according to frequency bands $\left(F_{(2,84)}=1575 ; p<0.0001 ; \eta_{p}^{2}=\right.$ $0.94)$, being significantly higher for $\delta-\theta$ compared with $\alpha-\sigma$ and $\beta-\gamma$ and for $\alpha-\sigma$ compared with $\beta-\gamma(p<0.0001)$ (Table 5). A single significant interaction was found between arousal and frequency band $\left(F_{(2,168)}=8.40 ; p=0.0003 ; \eta_{p}^{2}=0.09\right)$ (Table 6). Figure 3 shows that spectral power in slow waves was significantly lower when preceding arousal compared with nonarousal.

During PS, ANOVA on prestimulus spectral power did not show significant differences with respect to arousal $\left(F_{(1,87)}=1.52 ; p=0.22\right)$ or cerebral network $\left(F_{(4,87)}=0.211\right.$; $p>0.999)$. However, there was a significant effect of frequency band $\left(F_{(2,87)}=1715 ; p<0.0001 ; \eta_{p}^{2}=0.95\right)$, and a significant interaction between arousal and frequency band $\left(F_{(2,174)}=3.40 ; p=0.0355 ; \eta_{p}^{2}=0.04\right)$, explained by the fact that $\delta-\theta$ power was significantly higher preceding arousal (Fig. 3) (for details, see Tables 5-7).

\section{Logistic regression}

Variables significantly associated with the arousal/nonarousal factor in ANOVA were introduced in a logistic regression model, to investigate whether a combination of explanatory variables would best classify iEEG prestimulus periods preceding arousal. When several putative regressors showed significant intercorrelation, their values were averaged and collapsed into a single variable. This was the case for the phase-coherence levels of the three frequency bands in both N2 sleep and PS. The final variables introduced in the model were spectral phase-coherence between the posterior insula and other networks (averaged across frequencies) and the $\delta-\theta$ spectral power. In N2 sleep, the model yielded a correlation coefficient of $R=0.421$ and significant odds ratios for both spectral power $\left(\mathrm{OR}=0.929 ; \chi^{2}: 11.63 ; 95 \% \mathrm{CI}\right.$ : $0.890-0.969 ; p=0.0007)$ and phase-coherence levels $(\mathrm{OR}=1.063$; $\chi^{2}: 9.22 ; 95 \%$ CI: $\left.1.022-1.106 ; p=0.0024\right)$. In PS, the logistic model yielded a similar correlation coefficient of $R=0.443$ but retained only one significant predictor of arousal, namely, a prestimulus enhanced phase-coherence level $\left(\mathrm{OR}=1.071 ; \chi^{2}: 134.75\right.$; CI: $1.033-1.111 ; p=0.0002)$.

\section{Discussion}

Prestimulus functional connectivity between the sensory posterior insula and higher-order networks was a significant predictor of arousal to nociceptive stimuli during sleep, both in N2 stage and in PS. In addition, prestimulus attenuation of slow-wave activity was also, and independently, associated with arousal, but only in N2 sleep. Two characteristics of iEEG activity appear therefore to influence the ability of a noxious stimulus to provoke an arousal: one being the variation of a global level of activation, indexed by the spectral power; and the other being the functional interaction between sensory and high-order cerebral structures, indexed by their phase-coherence. The influence of these two parameters on arousal appeared largely independent from each other, as suggested by the lack of mutual correlation. All in all, the present results suggest that during sleep, as during wakefulness, the prestimulus activation and information exchange among brain areas are instrumental in determining the level of processing of a noxious event (Boly et al., 2007; Ploner et
Table 7. Post hoc analyses of spectral power values with respect to the significant interaction between cerebral networks and frequency bands in PS $\left(F_{(8,174)}=6.13 ; p<0.0001\right)^{a}$

\begin{tabular}{|c|c|}
\hline Areas & PS \\
\hline$\delta-\theta$ & $\begin{array}{l}\text { senso vs ce: } t_{(33)}=4.23 ; p=0.0016 ; d=0.98 \\
\text { senso vs sa: } t_{(35)}=1.06 ; p=0.65 \\
\text { senso vs in: } t_{(35)}=1.94 ; p=0.35 \\
\text { senso vs em: } t_{(33)}=0.23 ; p=0.82 \\
\text { ce vs sa: } t_{(33)}=3.16 ; p=0.0268 ; d=0.66 \\
\text { ce vs in: } t_{(33)}=1.70 ; p=0.40 \\
\text { ce vs em: } t_{(33)}=4.33 ; p=0.0013 ; d=0.98 \\
\text { sa vs in: } t_{(35)}=0.80 ; p=0.68 \\
\text { sa vs em: } t_{(33)}=1.44 ; p=0.40 \\
\text { in vs em: } t_{(33)}=1.85 ; p=0.37\end{array}$ \\
\hline$\alpha-\sigma$ & $\begin{array}{l}\text { senso vs ce: } t_{(33)}=5.12 ; p=0.0001 ; d=1.08 \\
\text { senso vs sa: } t_{(35)}=1.84 ; p=0.26 \\
\text { senso vs in: } t_{(35)}=2.87 ; p=0.04 ; d=0.66 \\
\text { senso vs em: } t_{(33)}=1.55 ; p=0.35 \\
\text { ce vs sa: } t_{(33)}=2.77 ; p=0.045 ; d=0.59 \\
\text { ce vs in: } t_{(33)}=0.77 ; p=0.44 \\
\text { ce vs em: } t_{(33)}=5.99 ; p<0.0001 ; d=1.46 \\
\text { sa vs in: } t_{(35)}=1.20 ; p=0.42 \\
\text { sa vs em: } t_{(33)}=3.21 ; p=0.02 ; d=0.79 \\
\text { in vs em: } t_{(33)}=4.07 ; p=0.002 ; d=0.91\end{array}$ \\
\hline$\beta-\gamma$ & $\begin{array}{l}\text { senso vs ce: } t_{(33)}=5.11 ; p=0.0001 ; d=1.18 \\
\text { senso vs sa: } t_{(35)}=1.48 ; p=0.52 \\
\text { senso vs in: } t_{(35)}=2.43 ; p=0.12 \\
\text { senso vs em: } t_{(33)}=0.46 ; p=0.68 \\
\text { ce vs sa: } t_{(33)}=4.24 ; p=0.0014 ; d=0.99 \\
\text { ce vs in: } t_{(33)}=3.99 ; p=0.0025 ; d=0.83 \\
\text { ce vs em: } t_{(33)}=4.35 ; p=0.0011 ; d=1.16 \\
\text { sa vs in: } t_{(35)}=1.07 ; p=0.64 \\
\text { sa vs em: } t_{(33)}=0.79 ; p=0.68 \\
\text { in vs em: } t_{(33)}=1.53 ; p=0.52\end{array}$ \\
\hline
\end{tabular}

$\overline{{ }^{a} \text { senso, Sensory; ce, central executive; sa, salience; in, integrative; em, emotional. } t, p \text {, and } d \text { values if }}$ significant.

al., 2010; Wiech et al., 2010), which in this study was reflected by transient sleep disruption and arousal.

The mechanisms involved in this processing may differ in sleep and wakefulness. During waking state, prestimulus changes influencing perception have been related to attention, anticipation, and/or anxiety (Boly et al., 2007; Ploner et al., 2010; Wiech et al., 2010), factors that can hardly be invoked during sleep. In addition, metabolic studies suggested that perceiving stimuli as subjectively painful may depend on the regional activation in anterior insula and anterior/mid cingulate (Boly et al., 2007; Ploner et al., 2010; Wiech et al., 2010), and/or prestimulus EEG phasecoherence between parasylvian and anterior cingulate regions (Ohara et al., 2008). During sleep, however, we did not find any specific network to be precisely associated to the occurrence of an arousal reaction; instead, our results suggest a global phenomenon of increased functional connectivity between sensory and different high-level processing areas, including salience, cognitive, and limbic networks. The phenomenon observed in this study suggests that arousal tends to occur when sensory receiving areas are in a state of activity favorable to send information to other networks involved in stimulus processing, and/or when such networks (including executive, integrative, and emotionrelated areas) are in a favorable state of receptiveness to process input from sensory cortices (Bastuji et al., 2016; Garcia-Larrea and Bastuji, 2018).

During slow-wave sleep, electrophysiological activity fluctuates because of the intrinsic tendency of cortical neurons to fall into a so-called "down-state" after transient activation 
(Massimini et al., 2012; Pigorini et al., 2015). Such "down," or hypoactive, states are associated with an increasing density of slow waves, and in accordance our results showed a lower probability of arousal from external stimuli when N2 sleep iEEG exhibits such slow-wave-laden periods. This is consistent with the increased arousal thresholds associated with enhanced density of $\delta$ activity during N2 sleep (Neckelmann and Ursin, 1993), and with the inverse relationship between higher slow-wave density and lower reactivity to external stimuli during general anesthesia (Mhuircheartaigh et al., 2010, 2013).

Of notice, while both $\delta$ power and phase-coherence were significant predictors of arousal in N2 sleep, only phase-coherence reached significance in paradoxical sleep. Since slow-wave activity is notoriously low during PS, the absence of predictive value might be due in part to a poor estimation of $\delta$ power in this sleep stage. Conversely, prestimulus phase-coherence between sensory and high-order cerebral areas remained a significant predictor of nociceptive-related arousal during PS, as it was in N2 sleep. To the best of our knowledge, changes in preactivation and functional connectivity preceding external stimuli have never been systematically investigated in sleep. One single-case study, however, reported that noxious stimuli during PS induced behavioral responses (a distal limb motor action) only if stimuli were applied during a state of preactivation in a vast array of structures, including premotor, prefrontal, cingulate, and pre-supplementary motor area cortices, as well as hippocampus (Mazza et al., 2014). Notwithstanding the differences with the present study, these results concur in underscoring the importance of the state of the brain at the moment of stimulus reception in determining the stimulus capacity to trigger an arousal reaction, permit an oriented response, and, eventually, gain access to consciousness. According to some models of conscious perception (e.g., Damasio, 1998), a "core consciousness" is incessantly generated relative to any object with which an organism interacts. This transient sense of knowing (or "core self") rapidly vanishes unless a higher level of encoding is engendered which allows conscious recollection and requires conventional memory. The arousal reactions induced in the present study were in general short-lasting, rarely recalled on awakening next day, and hence unlikely to entail a level of full consciousness. Yet, they represent a model that helps understanding the functional determinants allowing, or blocking, the processing of intruding stimuli during sleep, including in pathologic conditions, such as chronic pain, where such stimuli tend to interrupt sleep continuity (Bjurstrom and Irwin, 2016; Gandhi et al., 2020).

\section{Limitations and perspectives}

Our results were obtained in epileptic patients, that is, subjects with putatively abnormal episodic brain function, which may represent a bias. All these patients, however, had nociceptive thresholds in the normal range, and their intracranial responses were similar to those obtained on surface recordings with source reconstruction in control subjects (Bradley et al., 2017). In accordance, the spectral content of sleep iEEG activity in these patients was not different from that obtained on surface EEG in healthy volunteers (Weiss et al., 2011). Other limitations derive from the fact that functional links determined through phase-coherence analysis do not prove direct causal influences of one structure on another, and we cannot exclude that they may result from functional connections through intermediary regions not recorded here (Liu et al., 2011). The data we present, although extensive, could not provide a full view of all cortical regions responding to noxious input; in particular, areas of the temporal lobe were lacking because of their frequent involvement in the epileptic process.

N2 and PS are not homogeneous states but have phasic and tonic components, which might affect differentially stimulus processing and were not discriminated in this study. We did not perform specific comparisons between substages to reduce the number of repeated tests and keep statistical power. Furthermore, we have previously shown that phasic N2 periods (i.e., containing spindles) have no differential effects on nociceptive arousal relative to tonic periods (Claude et al., 2015), and that responses in paradoxical sleep do not change between tonic and phasic periods (Bastuji et al., 2008).

We did not extend our coherence analysis to high- $\gamma$ ranges since phase synchronization of high- $\gamma$ activity appears restricted to small interareal distances and declines dramatically for longer ranges, whereas coherence in $\delta, \theta$, and $\alpha$ bands remains significant (e.g., von Stein and Sarnthein, 2000). Since our coherence analysis focused on widely spaced networks, we opted to concentrate on frequency ranges more likely to convey distant information (Lisman and Jensen, 2013).

Finally, although our study was focused on the nociceptive system (and hence used the posterior insula as a seed), one can suppose that the state of preactivation can determine, at least partially, the brain reactivity to other types of sensory stimuli as well, the main determinant of arousal being the functional relation between sensory and high-order networks, whatever the sensory system involved. Thus, in the case of auditory stimuli, arousal might be determined preferentially by coherence involving auditory sensory areas, rather than the posterior insula. Investigating this question may prove difficult, since auditory stimuli have in general little arousing effects unless they are of high intensity (Bastuji and Lavigne, 2016), which would make them potentially nociceptive and hence nonspecific. Using auditory stimuli with intrinsic significance for the sleeper might be a possible solution.

In conclusion, the results of the present study indicate that the level of brain interareal communication immediately before a noxious stimulus may determine the behavioral responsiveness to the incoming input, by facilitating or preventing the transfer of information from sensory to multiple higher-level cortical networks.

\section{References}

Bastuji H, Lavigne G (2016) Pain perception during sleep. In: Pain and the conscious brain (Garcia-Larrea L, Jackson PL, eds), pp 61-74. Philadelphia: Wolter Kluwer.

Bastuji H, Perchet C, Legrain V, Montes C, Garcia-Larrea L (2008) Laser evoked responses to painful stimulation persist during sleep and predict subsequent arousals. Pain 137:589-599.

Bastuji H, Mazza S, Perchet C, Frot M, Mauguière F, Magnin M, GarciaLarrea L (2012) Filtering the reality: functional dissociation of lateral and medial pain systems during sleep in humans. Hum Brain Mapp 33:26382649.

Bastuji H, Frot M, Perchet C, Magnin M, Garcia-Larrea L (2016) Pain networks from the inside: spatiotemporal analysis of brain responses leading from nociception to conscious perception. Hum Brain Mapp 37:43014315.

Bjurstrom MF, Irwin MR (2016) Polysomnographic characteristics in nonmalignant chronic pain populations: a review of controlled studies. Sleep Med Rev 26:74-86.

Boly M, Balteau E, Schnakers C, Degueldre C, Moonen G, Luxen A, Phillips C, Peigneux P, Maquet P, Laureys S (2007) Baseline brain activity fluctuations predict somatosensory perception in humans. Proc Natl Acad Sci USA 104:12187-12192. 
Bradley C, Bastuji H, Garcia-Larrea L (2017) Evidence-based source modeling of nociceptive cortical responses: a direct comparison of scalp and intracranial activity in humans. Hum Brain Mapp 38:4301-4315.

Chouchou F, Pichot V, Barthélémy JC, Bastuji H, Roche F (2014) Cardiac sympathetic modulation in response to apneas/hypopneas through heart rate variability analysis. PLoS One 9:e86434.

Claude L, Chouchou F, Prados G, Castro M, De Blay B, Perchet C, GarciaLarrea L, Mazza S, Bastuji H (2015) Sleep spindles and human cortical nociception: a surface and intracerebral electrophysiological study. J Physiol 593:4995-5008.

Cruccu G, Aminoff MJ, Curio G, Guerit JM, Kakigi R, Mauguiere F, Rossini PM, Treede RD, Garcia-Larrea L (2008) Recommendations for the clinical use of somatosensory-evoked potentials. Clin Neurophysiol 119:1705-1719.

Damasio AR (1998) Investigating the biology of consciousness. Philos Trans R Soc Lond B Biol Sci 353:1879-1882.

Doucet GE, Lee WH, Frangou S (2019) Evaluation of the spatial variability in the major resting-state networks across human brain functional atlases. Hum Brain Mapp 40:4577-4587.

Dum RP, Levinthal DJ, Strick PL (2009) The spinothalamic system targets motor and sensory areas in the cerebral cortex of monkeys. J Neurosci 29:14223-14235.

Frot M, Mauguière F (2003) Dual representation of pain in the operculo-insular cortex in humans. Brain 126:438-450.

Gandhi W, Pomares FB, Naso L, Asenjo JF, Schweinhardt P (2020) Neuropathic pain after thoracotomy: tracking signs and symptoms before and at monthly intervals following surgery. Eur J Pain 24:1269-1289.

Garcia-Larrea L, Bastuji H (2018) Pain and consciousness. Prog Neuropsychopharmacol Biol Psychiatry 87:193-199.

Garcia-Larrea L, Perchet C, Creac'h C, Convers P, Peyron R, Laurent B, Mauguière F, Magnin M (2010) Operculo-insular pain (parasylvian pain): a distinct central pain syndrome. Brain 133:2528-2539.

Guénot M, Isnard J, Ryvlin P, Fischer C, Ostrowsky K, Mauguiere F, Sindou M (2001) Neurophysiological monitoring for epilepsy surgery: the Talairach SEEG method. StereoElectroEncephaloGraphy. Indications, results, complications and therapeutic applications in a series of 100 consecutive cases. Stereotact Funct Neurosurg 77:29-32.

Isnard J, Taussig D, Bartolomei F, Bourdillon P, Catenoix H, Chassoux F, Chipaux M, Clémenceau S, Colnat-Coulbois S, Denuelle M, Derrey S, Devaux B, Dorfmüller G, Gilard V, Guenot M, Job-Chapron AS, Landré E, Lebas A, Maillard L, McGonigal A, et al. (2018) French guidelines on stereoelectroencephalography (SEEG). Neurophysiol Clin 48:5-13.

La Cesa S, Di Stefano G, Leone C, Pepe A, Galosi E, Alu F, Fasolino A, Cruccu G, Valeriani M, Truini A (2018) Skin denervation does not alter cortical potentials to surface concentric electrode stimulation: a comparison with laser evoked potentials and contact heat evoked potentials. Eur J Pain 22:161-169.

Lavigne G, Zucconi M, Castronovo C, Manzini C, Marchettini P, Smirne S (2000) Sleep arousal response to experimental thermal stimulation during sleep in human subjects free of pain and sleep problems. Pain 84:283290.

Lavigne G, Brousseau M, Kato T, Mayer P, Manzini C, Guitard F, Monplaisir J (2004) Experimental pain perception remains equally active over all sleep stages. Pain 110:646-655.

Lisman JE, Jensen O (2013) The $\theta-\gamma$ neural code. Neuron 77:1002-1016.

Liu CC, Shi CQ, Franaszczuk PJ, Crone NE, Schretlen D, Ohara S, Lenz FA (2011) Painful laser stimuli induce directed functional interactions within and between the human amygdala and hippocampus. Neuroscience 178:208-217.

Magnin M, Bastuji H, Garcia-Larrea L, Mauguière F (2004) Human thalamic medial pulvinar nucleus is not activated during paradoxical sleep. Cereb Cortex 14:858-862.
Magnin M, Rey M, Bastuji H, Guillemant P, Mauguière F, Garcia-Larrea L (2010) Thalamic deactivation at sleep onset precedes that of the cerebral cortex in humans. supp info. Proc Natl Acad Sci USA 107:3829-3833.

Massimini M, Ferrarelli F, Sarasso S, Tononi G (2012) Cortical mechanisms of loss of consciousness: insight from TMS/EEG studies. Arch Ital Biol 150:44-55.

Mazza S, Magnin M, Bastuji H (2012) Pain and sleep: from reaction to action. Neurophysiol Clin 42:337-344.

Mazza S, Perchet C, Frot M, Michael GA, Magnin M, Garcia-Larrea L, Bastuji H (2014) Asleep but aware? Brain Cogn 87:7-15.

Mazzola L, Isnard J, Mauguiere F (2006) Somatosensory and pain responses to stimulation of the second somatosensory area (SII) in humans: a comparison with SI and insular responses. Cereb Cortex 16:960-968.

Mhuircheartaigh RN, Rosenorn-Lanng D, Wise R, Jbabdi S, Rogers R, Tracey I (2010) Cortical and subcortical connectivity changes during decreasing levels of consciousness in humans: a functional magnetic resonance imaging study using propofol. J Neurosci 30:9095-9102.

Mhuircheartaigh RN, Warnaby C, Rogers R, Jbabdi S, Tracey I (2013) Slowwave activity saturation and thalamocortical isolation during propofol anesthesia in humans. Sci Transl Med 5:208ra148.

Neckelmann D, Ursin R (1993) Sleep stages and EEG power spectrum in relation to acoustical stimulus arousal threshold in the rat. Sleep 16:467477.

Neugebauer V, Galhardo V, Maione S, Mackey SC (2009) Forebrain pain mechanisms. Brain Res Rev 60:226-242.

Nunez PL, Srinivasan R, Westdorp AF, Wijesinghe RS, Tucker DM, Silberstein RB, Cadusch PJ (1997) EEG coherency: I. Statistics, reference electrode, volume conduction, Laplacians, cortical imaging, and interpretation at multiple scales. Electroencephalogr Clin Neurophysiol 103:499_ 515.

Ohara S, Crone NE, Weiss N, Kim JH, Lenz FA (2008) Analysis of synchrony demonstrates that the presence of "pain networks" prior to a noxious stimulus can enable the perception of pain in response to that stimulus. Exp Brain Res 185:353-358.

Ostrowsky K, Magnin M, Ryvlin P, Isnard J, Guénot M, Mauguière F (2002) Representation of pain and somatic sensation in the human insula: a study of responses to direct electrical cortical stimulation. Cereb Cortex 12:376-385.

Perchet C, Frot M, Charmarty A, Flores C, Mazza S, Magnin M, GarciaLarrea L (2012) Do we activate specifically somatosensory thin fibres with the concentric planar electrode? A scalp and intracranial EEG study. Pain 153:1244-1252.

Pigorini A, Sarasso S, Proserpio P, Szymanski C, Arnulfo G, Casarotto S, Fecchio M, Rosanova M, Mariotti M, Lo Russo G, Palva JM, Nobili L, Massimini M (2015) Bistability breaks-off deterministic responses to intracortical stimulation during non-REM sleep. Neuroimage 112:105113.

Ploner M, Lee MC, Wiech K, Bingel U, Tracey I (2010) Prestimulus functional connectivity determines pain perception in humans. Proc Natl Acad Sci USA 107:355-360.

Rorden C, Brett M (2000) Stereotaxic display of brain lesions. Behav Neurol 12:191-200.

Talairach J, Bancaud J (1973) Stereotaxic approach to epilepsy: methodology of anatomo-functional stereotaxic investigations. Prog Neurol Surg 5:297-354.

von Stein A, Sarnthein J (2000) Different frequencies for different scales of cortical integration: from local gamma to long range alpha/theta synchronization. Int J Psychophysiol 38:301-313.

Weiss B, Clemens Z, Bódizs R, Halász P (2011) Comparison of fractal and power spectral EEG features: effects of topography and sleep stages. Brain Res Bull 84:359-375.

Wiech K, Lin CS, Brodersen KH, Bingel U, Ploner M, Tracey I (2010) Anterior insula integrates information about salience into perceptual decisions about pain. J Neurosci 30:16324-16331. 